\title{
Design of orientation stages for step and flash imprint lithography
}

\author{
B.J. Choi ${ }^{\text {a }}$, S.V. Sreenivasan ${ }^{\mathrm{a}, *}$, S. Johnson ${ }^{\mathrm{a}}$, M. Colburn ${ }^{\mathrm{b}}$, C.G. Wilson ${ }^{\mathrm{b}}$ \\ ${ }^{a}$ Department of Mechanical Engineering, University of Texas at Austin, Austin, TX 78712, USA \\ ${ }^{\mathrm{b}}$ Department of Chemical Engineering, University of Texas at Austin, Austin, TX 78712, USA
}

Received 2 November 1999; received in revised form 17 October 2000; accepted 18 December 2000

\begin{abstract}
This paper presents the design of orientation stages for high-resolution imprint lithography machines. These machines implement a new lithography process known as Step and Flash Imprint Lithography (SFIL) and are intended for 1) sub 100 nm imprint demonstrations on flat substrates and 2) investigation of potential defect propagation during step and repeat imprinting. SFIL is an imprint lithography process that is a combination of chemical and mechanical steps and its implementation at room temperature and low pressure makes it an attractive process as compared to other imprint techniques. A critical component of an imprint machine is the orientation stage that is required to provide uniform intimate contact between the template and substrate surfaces. The orientation stage requirements are distinct from those used in photolithography since the depth of focus of projection optics allows for larger errors in the orientation alignment. Also, due to contact between the template and substrate surfaces in imprint lithography, the separation kinematics must be carefully controlled in the SFIL process. Two different orientation stages are designed for single- and multi-imprint machines. In order to eliminate the particle contamination due to frictional contacts, all joints are made with flexure joints. Imprint experiments have been performed to demonstrate sub 100 nm imprints. (C) 2001 Elsevier Science Inc. All rights reserved.
\end{abstract}

Keywords: Template-wafer alignment; Template stage; Flexure mechanism; Step and Flash Imprint Lithography

\section{Introduction}

Step \& Flash Imprint Lithography (SFIL) has been introduced as a low cost approach that can potentially generate circuit patterns with sub $100 \mathrm{~nm}$ line width without expensive projection optics [1,2]. Imprinting on curved substrates has also been demonstrated using templates patterned by Ion Beam Proximity Printing [3]. SFIL is a room temperature and low pressure process that largely relies on chemical and mechanical steps to transfer high resolution patterns. One could probably best describe SFIL as a micro-molding process (See [4-8] for other imprint processes under development). SFIL and other imprint lithography techniques are similar in the fact that they use the topography of a template to define the pattern created on the substrate. The key difference between SFIL and other imprint lithography techniques is the use of a liquid etch barrier. This low viscosity solution eliminates the need for high temperatures and pres-

$$
8727 .
$$

* Corresponding author. Tel.: +1-512-471-6546; fax: +1-512-471-

E-mail address: sv.sreeni@mail.utexas.edu (S.V. Sreenivasan). sures. High temperatures and pressures can cause major technical problems in accurate overlaying of multiple layers of a circuit and are hence undesirable. Fig. 1 illustrates the SFIL process [1]. Since it is impractical to have a zero gap between the template and substrate, the imprinted feature on the substrate surface has a thin film of etch barrier and this layer is called the base layer in Fig. 1. For a practical imprint lithography process, the base layer needs to be thin (usually $100 \mathrm{~nm}$ or less) and uniform across the imprinted area. The maximum thickness variation of the base layer needs to be less than the height of the imprinting feature for a successful etching process. Fig. 2 illustrates relative configurations of template and substrate that lead to undesirable base layers.

\section{Imprint machines}

Two imprint machines have been developed for the feasibility study of the SFIL process. Fig. 3 shows a schematic diagram for the SFIL process. A single-imprint machine was developed to investigate the resolution limit of the SFIL 


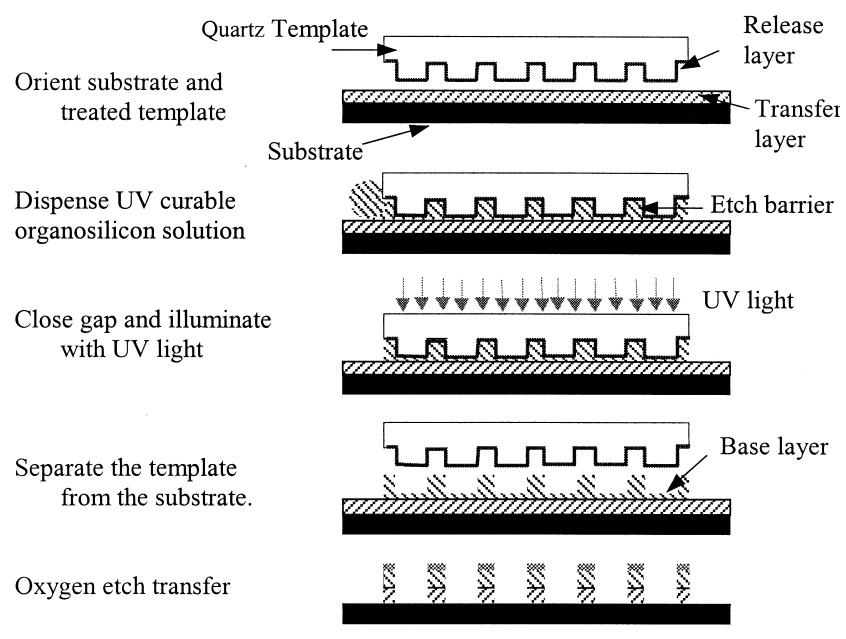

Fig. 1. The Step and Flash Imprint Lithography process.

process and to support the development of materials associated with the transfer layer and the etch barrier. A multiimprint machine that can perform repeated imprints on 8 inch wafers was developed for the purpose of defect propagation analysis [9]. It can also provide an accurate control of the base layer to enable the oxygen etch transfer. The major machine components of the multi-imprint machine include the following: (a) A micro-resolution Z-stage that can control the average distance between the template and substrate, and can control the imprinting force; (b) An automated XY-stage for the step and repeat positioning; (c) A pre-calibration stage that assists in obtaining parallel alignment between the template and substrate by compensating for orientation errors introduced during the template installation; (d) A vacuum chuck that has a uniform distribution of vacuum applied to the bottom of the substrate and that has a carefully polished surface to ensure that the substrate has low topography variations (is flat to within a few tens of nanometers); (e) A fine-orientation flexure stage that augments the alignment capability of the pre-calibration stage to provide a highly accurate parallel alignment of the template and substrate; the alignment accuracy is of the order of tens of nanometers across an inch; (f) A flexure based substrate calibration stage that orients the top of the substrate surface parallel with respect to the plane of the XY-stage; (g) An exposure source that is used to cure the etch barrier; (h) An automated fluid delivery system that can accurately dispense known amounts of the liquid etch barrier; and (i) Load cells that can provide both imprinting and separation force data.

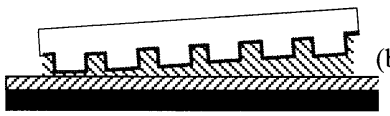

(b)

Fig. 2. Relative positioning of the template and substrate that leads to undesirable base layers: (a) a wedge shaped base layer and (b) a base layer that is too thick.

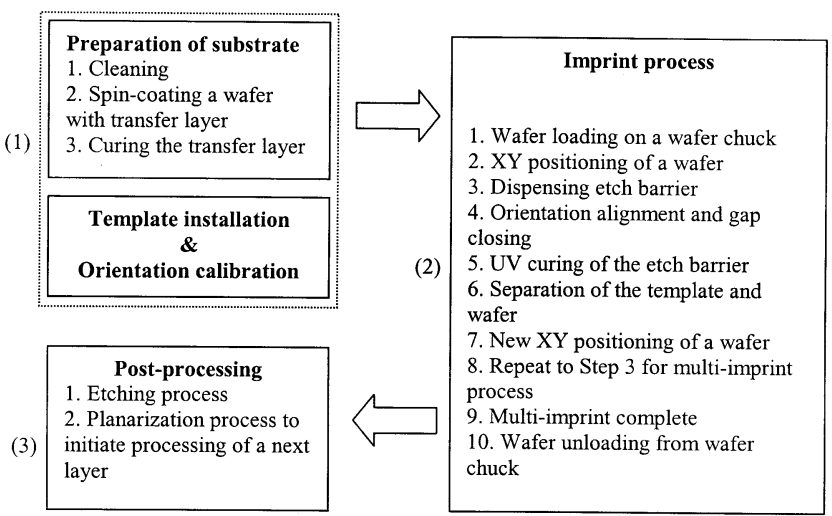

Fig. 3. The SFIL process flow associated with multi-imprinting. A singleimprint process includes only steps 1 through 6 .

A critical mechanical issue for these imprint machines is the fine orientation stage that must bring the template and substrate surfaces into contact while minimizing relative lateral motions. In this work, passive compliant orientation stages that generate their motions by pushing the template against the substrate were developed. The stages were, however, designed keeping in mind that they can be modified to make them piezo-actuated for future research. The need to minimize particle generation from orientation stages and other moving bodies is important since particle contamination can be a major source of defect propagation in this process.

In this investigation, first, kinematic requirements of the orientation stages were studied (Section 3). Passive stages satisfying the kinematic requirements were developed next using flexure mechanisms. Presence of sliding contacts in mechanical joints can cause wear, generate undesirable particles and lead to stiction that makes precision motion control difficult. Presence of clearances in joints can lead to reduced repeatability in the motion of the mechanism.

Flexures generate motion by elastic deformation and can avoid all the problems associated with frictional joints. Also, provided elastic and fatigue limits are not exceeded, flexures can provide extremely repeatable motion and long life for the stage. Flexure stages are becoming quite common in the precision engineering industry $[10,11]$.

\section{Orientation stages for imprint machines}

Fig. 4 shows two flat surfaces representing the template and substrate surfaces respectively. A proper alignment between these two flats ideally leads to a perfectly uniform surface contact between them. Such an alignment can be accomplished with one translation ( $\mathrm{z}$ displacement) and two 


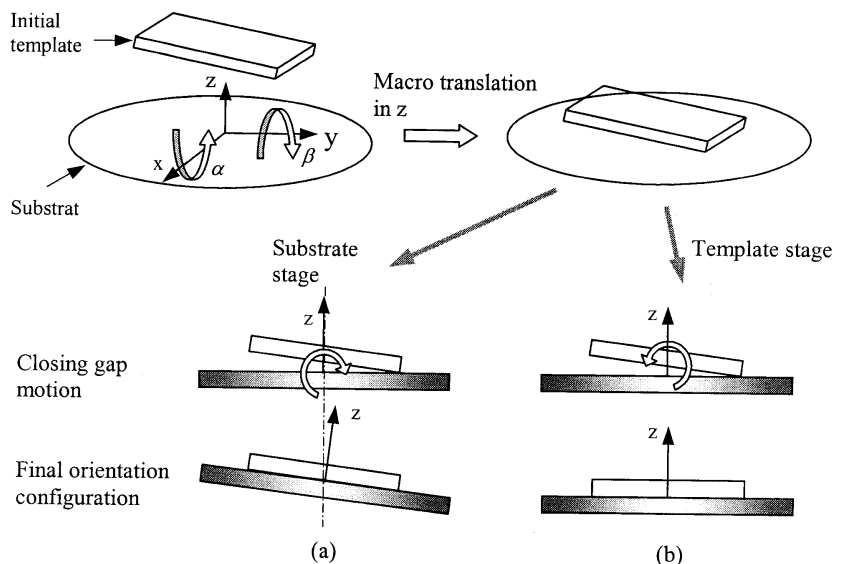

(a)

(b)

Fig. 4. Orientation alignment motions for (a) a substrate orientation stage and (b) a template orientation stage. $\mathrm{x}$ and $\mathrm{y}$ are axes are on the substrate surface.

tilting motions ( $\alpha$ and $\beta$ ) between two flats. The $\mathrm{x}$ and $\mathrm{y}$ axes are located on the substrate surface.

\subsection{Lateral motion error due to coupled tilting motions}

The three necessary orientation motions were identified above. In an ideal situation, the translation motions of a template in $\mathrm{x}$ and $\mathrm{y}$ (see Fig. 4 for the axes) should be eliminated. Fig. 5 shows a coupled effect between tilting and translation. Coupled motions arise when a tilting axis does not exist on the surface of the template. A lateral displacement the template, $d_{s}$, is given by $d_{s}=$ $l_{\text {off }} \sin \theta \cong l_{\text {off }} \theta$, (when $\theta$ is small) where, $l_{\text {off }}$ is the offset of the tilting axis from the surface and $\theta$ is the tilting angle in radians.

As an example, when $l_{\text {off }}=5 \mathrm{~mm}$ and $\theta=0.0001$ radian, $d_{s}$ is $500 \mathrm{~nm}$. Such an excessive lateral displacement of the template must be avoided for a high-resolution imprint process. During a separation process in which the etch

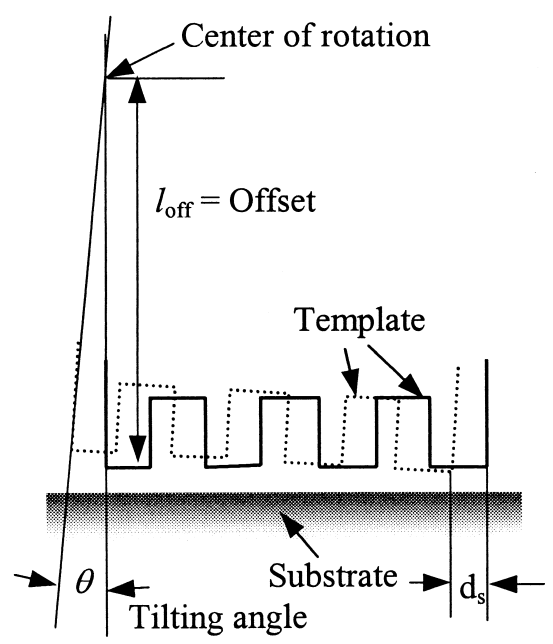

Fig. 5. Lateral motion of the template due to tilting about an offset axis.

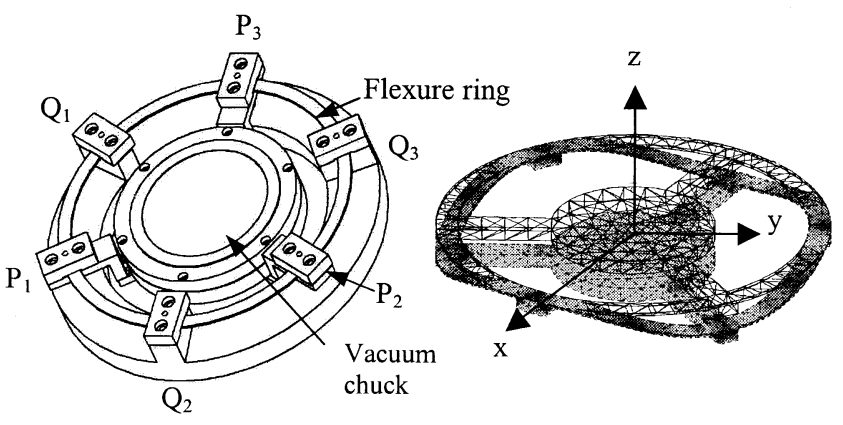

Fig. 6. Substrate orientation stage and a FEA analysis showing the deformed ring structure under a vertical load.

barrier has already been UV exposed, excessive lateral displacements may destroy transferred images.

\subsection{Orientation stage for single-imprint machine}

For the single-imprint machine, a substrate orientation stage has been developed [12]. The basic element of the flexure is derived from the work presented by Badami et al. [9]. They presented a ring that is comprised of three fixed-fixed beams. The mid-point of each of these beams (points $\mathrm{P}_{1}, \mathrm{P}_{2}$, and $\mathrm{P}_{3}$ in Fig. 6) provide a vertical deflection due to an applied vertical load. A modified version of this design yields the distributed flexure stage of Fig. 6. This three degree-of-freedom (DOF) stage was developed keeping in mind the need for making the stage actively controlled. A high resolution $\mathrm{z}$ directional gap control can be achieved in addition to orientation alignment by this flexure using piezo actuators attached at the points $\mathrm{P}_{\mathrm{i}}, \mathrm{i}=1,2$ and 3 .

The three Points, $\mathrm{P}_{\mathrm{i}}$, of the fixed-fixed beams are connected to a vacuum chuck. To avoid shearing due to relative motion between the template and substrate, the flexure ring is installed such that the orientation axes are aligned with the template and substrate interface. The parameters of the ring (material and geometry) can be arrived at by using the desired motion range for the $\mathrm{z}$-translation and the two tilting motions [12].

A numerical result from an FEA analysis is discussed next. No linear or rotational displacements are allowed at the three locations where the flexure ring connects to the base $\left(Q_{i}\right.$ in Fig. 6). These conditions simulate a rigid structure supporting the flexure ring. Six linearly independent forces and moments (each column of matrix $\mathbf{F}$ ) were applied to the structure. The top three elements of each column of matrix $\mathbf{F}$ denote linear forces and the lower three elements denote moments applied about the center of the vacuum chuck. The top three elements of each column of matrix $\mathbf{T}$ denote rotations about $\mathrm{x}-\mathrm{y}-\mathrm{z}$ axes and the lower three elements denote translations in the directions of $x-y-z$ axes. Fig. 6 shows the coordinate system used for the FEA model. 


$$
\begin{aligned}
& \mathbf{F}=\left\{\begin{array}{cccccc}
66.7 & 0 & 0 & 0 & 0 & 0 \\
0 & 66.7 & 0 & 0 & 0 & 0 \\
0 & 0 & -66.7 & 0 & 0 & 0 \\
0 & 0 & 0 & 0 & -1840.2 & 362.6 \\
0 & 0 & 0 & 0 & 124.10 & 1796.20 \\
0 & 0 & 0 & -1416.5 & 0 & 0
\end{array}\right\} \text { forces } \\
& \mathbf{T}=\left\{\begin{array}{cccccc}
0 & -0.0005 & 0 & 0 & -0.0116 & 0.0023 \\
0.0005 & 0 & 0.0016 & 0 & 0.0007 & 0.0101 \\
0 & 0 & 0 & -0.0001 & 0 & 0 \\
0.0197 & 0 & 0.0240 & 0 & 0.0013 & 0.0206 \\
0 & 0.0198 & 0 & -0.0005 & 0.0225 & -0.0045 \\
-0.0013 & 0.0001 & -1.4180 & 0 & 0.0011 & -0.0369
\end{array}\right\} \text { rotation }
\end{aligned}
$$

The information contained in $\mathbf{F}$ and $\mathbf{T}$ describes the resulting displacement $\mathbf{t}_{\mathbf{i}}$ for a set of six linearly independent force-moments $\mathbf{f}_{\mathbf{i}}$. The first column of $\mathbf{F}, \mathbf{f}_{\mathbf{1}}$, represents a $66.7 \mathrm{~N}(15 \mathrm{lb}$.) force applied along the $\mathrm{x}$ axis. The first column of $\mathbf{T}, \mathbf{t}_{\mathbf{1}}$, indicates that the stage undergoes a 0.0005 radian rotation about the $y$ axis and translates $0.0197 \mathrm{~mm}$ along the $\mathrm{x}$ axis. $\mathbf{f}_{2}$ represents a $66.7 \mathrm{~N}(15 \mathrm{lb}$.) force applied along the $\mathrm{y}$ axis. $\mathbf{t}_{\mathbf{2}}$ indicates that the stage undergoes a -0.0005 radian rotation about the $\mathrm{x}$ axis and translates $0.0198 \mathrm{~mm}$ along the y axis. $\mathbf{f}_{\mathbf{3}}$ represents a $-66.7 \mathrm{~N}(-15$ lb.) force applied along the $\mathrm{z}$ axis. $\mathbf{t}_{\mathbf{3}}$ indicates that the stage undergoes a 0.0016 radian rotation about the $y$ axis and translates $1.4 \mathrm{~mm}$ down the $\mathrm{z}$ axis. The remaining pairs may be interpreted in a similar manner.

Note that the stage is compliant about the $\mathrm{x}$ and $\mathrm{y}$ axes and along the $\mathrm{z}$ axis. The stage is most likely to rotate about $\mathrm{x}$ and $\mathrm{y}$ and translate in $\mathrm{z}$. Table 1 lists strain energies calculated from applied forces and displacements. For a given force (moment), the strain energies (or deflection) in $\mathrm{x}$ and $\mathrm{y}$, and rotation about $\mathrm{z}$ are two orders of magnitude lower than the strain energies in $\mathrm{z}$ and rotations about $\mathrm{x}$ and $y$. Small strain energies indicate that forces along the $x$ and $\mathrm{y}$ axes and moments about the $\mathrm{z}$ axis are supported by the flexure with small deflections. Compared to the small deflections along $\mathrm{x}$ and $\mathrm{y}$ and small rotation about $\mathrm{z}$, the flexure stage allows large rotations about $\mathrm{x}$ and $\mathrm{y}$ and translation in z. These results confirm that the distributed flexure stage exhibits the motions that are desired in a substrate orientation stage.

Table 1

Strain energy in flexure stage

\begin{tabular}{ll}
\hline Strain Energy & Applied wrench \\
\hline 1.3125 & $15 \mathrm{lb}$. force along $\mathrm{x}$ axis \\
1.3185 & $15 \mathrm{lb}$. force along $\mathrm{y}$ axis \\
94.6136 & $15 \mathrm{lb}$. force along $\mathrm{z}$ axis \\
119.0054 & $10 \mathrm{in}-\mathrm{lb}$. torque about $\mathrm{x}$ axis \\
116.1306 & $10 \mathrm{in}-\mathrm{lb}$. torque about $\mathrm{y}$ axis \\
1.4617 & $10 \mathrm{in}-\mathrm{lb}$. torque about $\mathrm{z}$ axis \\
\hline
\end{tabular}

\subsection{Template orientation stage for multi-imprint machine}

Multi-imprinting can be performed via moving a substrate to various positions while holding a template stationary in $\mathrm{x}$ and $\mathrm{y}$ directions. For the multi-imprint process, it is necessary to have a flexure based orientation alignment stage on the template side since a fixed template and compliant substrate stage can lead to unstable configuration for multiple $x-y$ positions of the substrate. For example, when the template and substrate are positioned as in Cases 1 and 2 of Fig. 7, passive compliant substrate stage will lead to a proper alignment between the template and substrate surfaces. When the template is in an off-centered position and is oriented as shown in Case 3, a passive compliant substrate stage can still align the template and substrate surfaces. However, for the Case 4, pushing the template downward will not align the two surfaces but increase the misalignment further (Fig. 8). Hence a new orientation stage design that can orient the template instead of the substrate is desired.

Another desired modification of the orientation alignment stage is to decouple the $\mathrm{z}$ translation from the two tilting motions. As described in Section 2, the average gap between the template and substrate can be controlled using a translation actuator. Therefore, instead of a three DOF

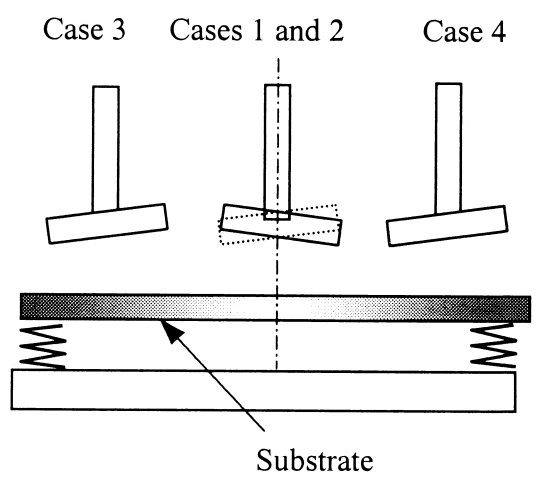

Fig. 7. Possible configurations of template and compliant substrate stage (a substrate stage works for Case 1,2, and 3 only). 


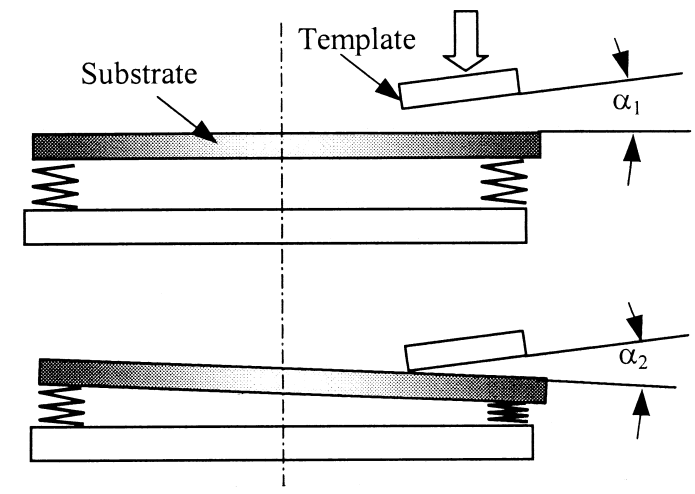

Fig. 8. Passive compliant substrate stage cannot correct for the initial orientation error in Case 4 of Figure $7\left(\alpha_{2}>\alpha_{1}\right)$.

flexure stage as shown in Fig. 6, a two DOF flexure stage that can passively correct the orientation error is pursued for the multi-imprint machine.

The template stages for the multi-imprint machine have been designed such that the above two modifications can be accommodated. Also, for a practical template stage, all mechanical components of the stage should be above the template in order to eliminate physical contact between the mechanical components and the substrate. Therefore, a template orientation stage that can tilt the template about "remote axes" was developed.

Fig. 9 shows a simple four-bar-linkage with ideal links and joints in its nominal and rotated configurations. The angle between the line passing through Joints 1 and 2 and the other line passing Joints 3 and 4 is selected so that the compliant alignment axis lies exactly on the templatesubstrate interface. For fine orientation changes, the rigid body between Joints 2 and 3 rotates about an axis that is depicted by Point $\mathrm{C}$ in Fig. 9 (the axis is normal to the paper). Since frictional contacts are undesirable for the imprint process due to particle contamination, all joints are made of flexure joints for the template orientation stage. Fig. 10 shows a schematic of a two DOF flexure

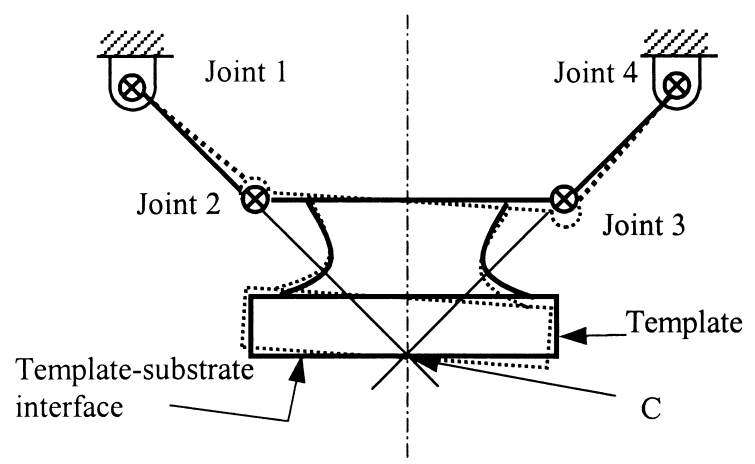

Fig. 9. Four bar linkage composed of ideal joints and links. Dotted line represents the rotated configuration for a small angular displacement.

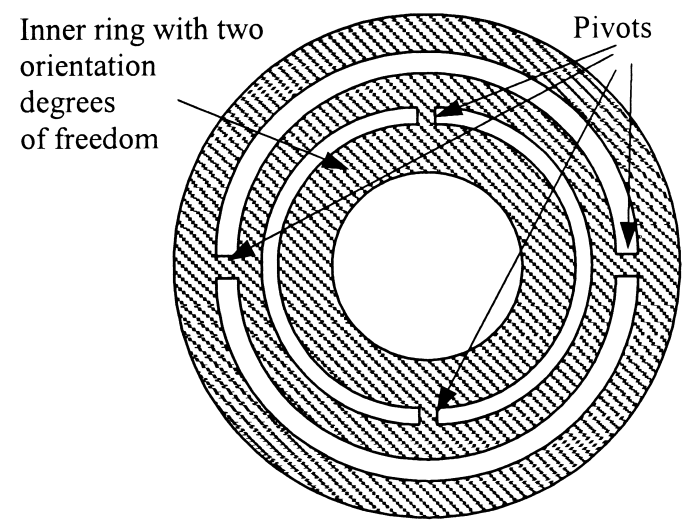

Fig. 10. Two degrees of freedom flexure stage.

stage $^{a}$ that is equivalent to two serially connected tilting mechanisms. The pivot can be made of I or H-beam structure so that its torsion stiffness can be significantly lower than the bending stiffness. This is a monolithic design that allows for both tilting motions. However, it is not suitable as a stage that supports the template because the tilting axes cannot be remotely located on the template surface.

Parameters of each semi-circular notch of the four bar flexures of Fig. 11 have been determined based on nominal vertical load and motion requirements. The spring coefficient of each semi-circular notch is desired to be low so that necessary orientation motions can be achieved with a low normal load between the template and substrate surfaces ${ }^{\mathrm{b}}$. For the imprint process, however, the template orientation stage should be able to support required imprinting loads. The geometry of the semi-circular notch is designed so that when a $4 \mathrm{~N}$ load is applied at a distance of $10 \mathrm{~mm}$ from the center, the stage rotates about 0.0005 radian (see Appendix).

A FEA analysis was performed to verify the selected design. Assuming a small initial orientation misalignment between the template and substrate, a localized external force is applied to represent a load at the edge of the template (here template is assumed to be $20 \mathrm{~mm}$ wide). Fig. 11 (b) shows a magnified lateral deformation (x direction) of the template; it does not include vertical (z) deformation. The results showed that the template surface moves less than $5 \mathrm{~nm}$ laterally when it undergoes a tilting of 0.00038 radian.

Fig. 12 shows the assembled configuration of a quartz template with flexure template stages. The second flexure component is mounted on the first one so that the two decoupled orientation axes are orthogonal to each other and they lie on the template surface. The flexure devices can be readily adapted to have open slots so that a curing UV light can pass through the template as required in SFIL process.

\footnotetext{
${ }^{\mathrm{a}}$ Fig. 10 was suggested by one of the reviewers of this article.

${ }^{b}$ Excessive loads may cause not only undesirable large deformations but also mechanical failure of either template or substrate.
} 


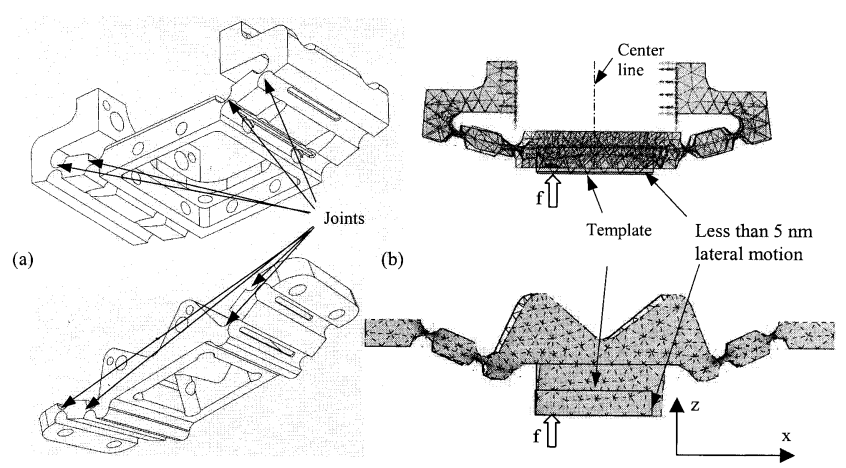

Fig. 11. (a) Flexure template stages, (b) lateral deformations of the template stage for a small rotation; an external force, $\mathrm{f}$, is applied at a distance of 10 $\mathrm{mm}$ from the center line in order to model an initial orientation misalignment between the template and substrate.

Prior to imprinting, two steps of calibrations are required. The first calibration is to achieve the coplanarity of the entire substrate with respect to the XY plane of the XY stage. To achieve this, the template is lowered by the same distance in three positions that are located near the periphery of the substrate and the imprinting forces are measured. Once the force is balanced the first calibration is completed. The second calibration is to compensate the installation errors of the template. The template is lowered at the center of the substrate until a small pressure (typically $0.50 \mathrm{psi}$ ) is measured. Using the three differential micrometers attached to flexure calibration stage (see Fig. 12), the orientation of the template is manually adjusted by observing the interference fringes that appear at the template and substrate interface. When the number of fringes drops below one, the template and substrate are in parallel to within $150 \mathrm{~nm}$ error across the template surface (when green light is used for interface pattern generation). This manual calibration is required only when a new template is installed. Any remaining small orientation errors between the template and substrate are corrected by the template flexure stages. Fig. 13 shows the multi-imprint machine.

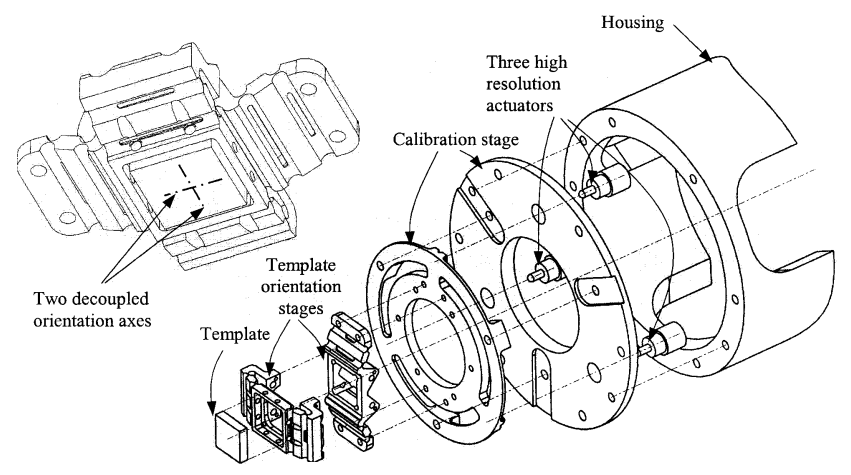

Fig. 12. Components of the orientation alignment stage for the multiimprint machine. Two template orientation stages are assembled with a template so that two decoupled orientation axes exist on the template surface.

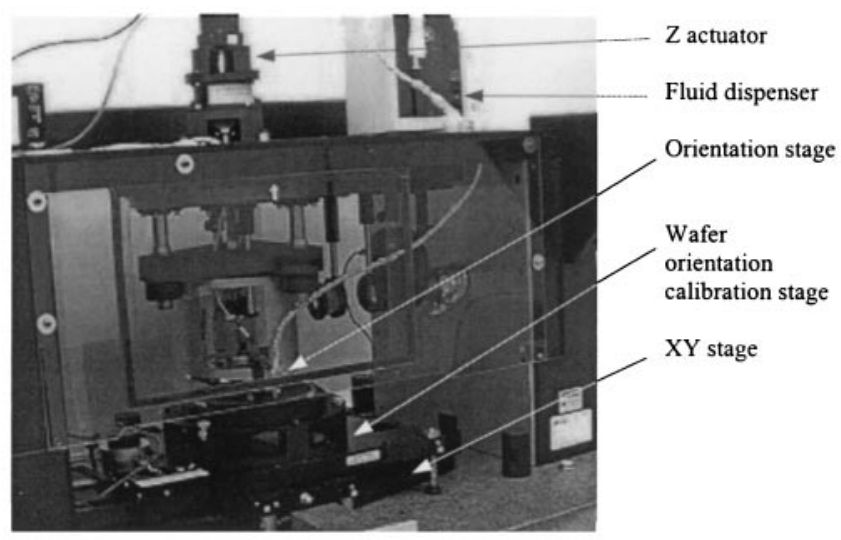

Fig. 13. Multi-imprint machine developed for the resolution and defect studies of SFIL.

\section{Imprint results}

Imprinted results using the Step and Flash Imprint Lithography process are presented here. The resolution limit and orientation capability under a low imprinting pressure were studied in this article.

Fig. 14 shows $60 \mathrm{~nm}$ features on the template and wafer surfaces. It has been observed that the SFIL process can duplicate not only such small features but also much smaller defects on the template such as stitching errors in the electron beam process that is used to fabricate the template. Hence, it is expected that the resolution limit SFIL is significantly smaller than $60 \mathrm{~nm}$. A SEM image of $150 \mathrm{~nm}$ features on the wafer surface is also shown in Fig. 14 to indicate that features have clearly defined side walls.

Fig. 15 shows imprinted images where 12 regions of features were imprinted. Fig. 15 (a) contains several fringe patterns across the imprinted area of 1 inch by 1 inch, which indicates that the orientation between the template and substrate was not properly compensated. Using the calibration stage, the template and wafer surfaces were calibrated to within one fringe across 1 inch by 1 inch that yields the imprinted area shown in Fig. 15 (b). The typical imprinting pressure was less than three psi for the results shown here.

\section{Conclusions}

Selectively compliant flexure stages have been designed for single- and multi-imprint lithography machines. It is

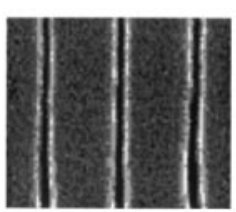

(a)

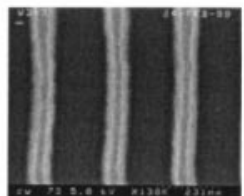

(b) (c)

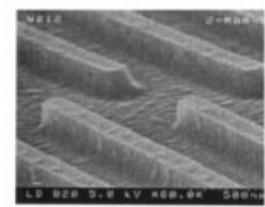

Fig. 14. $60 \mathrm{~nm}$ features (a) on the template and (b) on wafer; and (c) 150 $\mathrm{nm}$ features transferred to the etch barrier. 


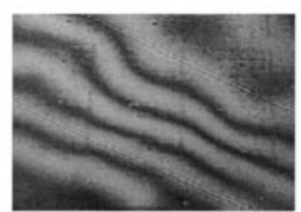

(a)

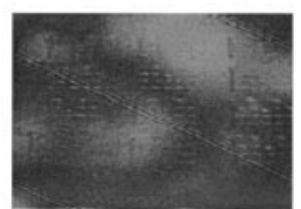

(b)

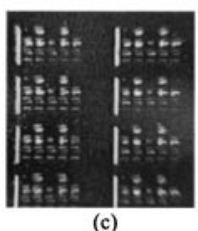

(c)
Fig. 15. Imprint results: (a) orientation misalignment leads to a transferred image with several fringe patterns; (b) less than one fringe pattern; (c) large area image (1 inch by 1 inch).

assured that the kinematics of the stages are consistent with the requirements of aligning two flats into intimate contact. Further, orientation axes are placed at the template-substrate interface to ideally eliminate lateral motion due to change in orientation angles. FEA analyses have been used to analyze the functionality of the flexure stages. The stages have been fabricated and tested in conjunction with Step and Flash Imprint Lithography. Sub $100 \mathrm{~nm}$ patterning on large area substrates has been successfully demonstrated. The orientation alignment between the template and substrate has been also successfully demonstrated using the calibration stage and the template orientation stage.

\section{Acknowledgment}

We gratefully acknowledge the financial support of DARPA (grant N66001-98-1-8914). We also appreciate the reviewers for their constructive comments.

\section{Appendix A. For a given force and the width of the joint $(b=0.0127 \mathrm{~m})$, compute the radius and the thickness of the thinnest section of the semi-circular notch.}

For a steady stage, the relationship between the external force $(f)$ and the rotation at each joint is given by (using virtual work),

$$
f \times \mathrm{d}_{\text {off }} \times \mathrm{d} \theta=\sum_{i=1}\left(k_{i} \times \theta_{i}\right) \mathrm{d} \theta, \text { where } k_{i} \text { is spring }
$$

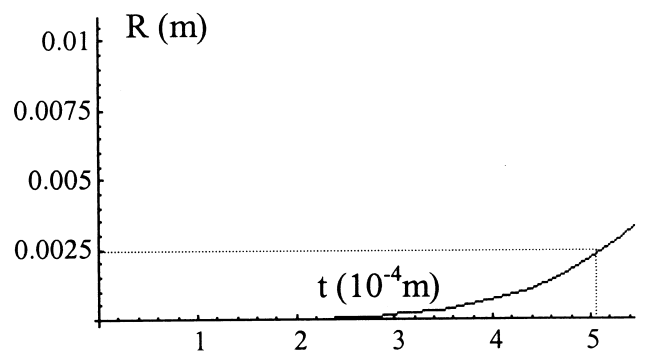

Fig. A-2. Radius (R) - thickness (t) for a given spring coefficient.

$$
\text { stiffness at joint } i=1,2,3,4 .
$$

Here, $\mathrm{d}_{\text {off }}=$ offset from the center of the template $=$ $0.01 \mathrm{~m}$. When we assume that each joint rotates about 0.0005 radian when $f=4 \mathrm{~N}$ (actually, Joint 2 and 3 rotate more than Joint 1 and 4 in Figure 11), $\mathrm{k}$ is given as,

$$
\begin{aligned}
k & =4 N \times 0.01 \mathrm{~m} /(4 \times 0.0005 \mathrm{rad}) \\
& =20(N-\mathrm{m} / \mathrm{rad}) .
\end{aligned}
$$

When $R \gg t$, the spring coefficient of semi-circular notch can be approximated as [PW65],

$$
k=\left(2 E b t^{5 / 2}\right) /\left(9 \pi R^{1 / 2}\right),
$$

where, $t$ is the thickness of the thinnest cross section and $R$ is the radius of the half circle. See Figure A-1 for the dimensions of the semi circular notches used for the flexure stages.

Fig. A-2 shows the relationship between the radius $(R)$ and the thickness of the thinnest cross section $(t)$ of the semi-circular notch for a given $\mathrm{k}$. Based on this plot, $R=$ $2.5 \mathrm{~mm}$ and $t=0.5 \mathrm{~mm}$ are selected for the semi-circular notches.

Once the notch geometry is determined, the normal stress and bending stress due to the applied load are computed. Excessive normal or bending stresses of the flexure linkage should be avoided.

- With an applied load: $f_{N}=10 \mathrm{~N}$ (at the center of the template)

- Incline angle of the linkage: $\theta=16$ Degrees (the angle between Joints 4-1-2 of Fig. 9)

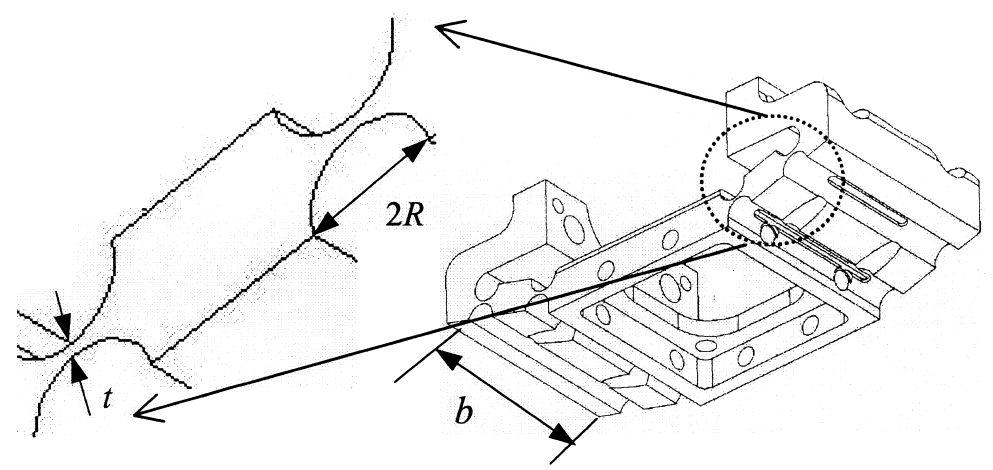

Fig. A-1. Three dimensions for the semi-circular notches. 
1. Normal stress at notch $=20 / \sin \left(16^{\circ}\right) /(t \times 0.0127)=$ $2856.6 / t$ when $t=0.5 \mathrm{~mm}, \sigma_{\text {normal }}=5.6 \mathrm{Mpa}$

2. Ignoring the stress concentration at the outer edges at each end of the flexure, at the onset of yielding, the stress in such a notch for a given maximum angular displacement is given by

$$
\begin{aligned}
\sigma_{\text {bending }}= & (E t) /(4 R) \theta_{\max }=200 \mathrm{Gpa} \times 0.0005 \\
& \times 0.001 /(4 \times 0.0025)=10 \mathrm{Mpa}
\end{aligned}
$$

Therefore, both stresses are well below the yield stress (for the case of $R=2.5 \mathrm{~mm}$ and $t=0.5 \mathrm{~mm}$ ). The safety factor of the flexure notch is estimated to be 10 approximately.

\section{References}

[1] Colburn M, et al. Step and Flash Imprint Lithography: A Novel Approach to Imprint Lithography, SPIE's $24^{\text {th }}$ Annual International Symposium on Microlithography, Santa Clara, CA, 1999.

[2] Ruchhoeft P, et al. Patterning Curved Surfaces: Template Generation by Ion Beam Proximity Lithography and Relief Transfer by Step and Flash Imprint Lithography, J. Vac. Sci. Technol. Nov. 1999.

[3] Stumbo DP, et al. J Vac Sci Technol 1991;B9:3597.

[4] Chou SY, et al. Nanoimprint Lithography, J Vac Sci Technol B 1996;14(6):4129-33.

[5] Haisma J, et al. Template-assisted Nanolithography: A Process for Reliable Pattern Replication. J Vac Sci Technol B 1996;14(6):412429.

[6] Wang D, et al. Nanometer Scale Patterning and Pattern Transfer on Amorphous Si, Crystalline Si, and Sio2 Surface using Self-Assembled Monomlayers. Appl Phys Lett 1997;70:12.

[7] Widden TK, et al. Pattern Transfer to Silicon by Microcontact Printing and RIE. Nanotechnology 1996;7:447-51.

[8] Xia Y, Whitesides GM, Soft Lithography. Angew Chem Int 1998; 37:550-75.

[9] Badami VG, et al. A Metrological Three-Axis Translator and Its Application for Constant Force Profilometry. Proceedings of ASPE 1996 Annual Meeting, 1996. p. 391-5.

[10] Paros JM, Weisbord L. How to Design Flexure Hinges. Machine Design 1998, No T-27, pp. 151-6.

[11] Smith S, Chetwynd DG. Foundations of Ultraprecision Mechanism Design. Gordon and Breach Science Publishers, Philadelphia, 1992.

[12] Johnson S. Selectively Compliant Orientation Stages for Imprint Lithography. MS thesis, The University of Texas at Austin, 1999. 